\title{
Effectiveness of Fluorescence-Based Methods to Detect in situ Demineralization and Remineralization on Smooth Surfaces
}

\author{
C.M. Moriyama ${ }^{a} \quad$ J.A. Rodrigues ${ }^{b} \quad$ A. Lussic $^{c}$ M.B. Diniz ${ }^{a}$ \\ a Department of Pediatric Dentistry, School of Dentistry, Cruzeiro do Sul University, UNICSUL, São Paulo, and \\ ${ }^{b}$ Department of Pediatric Dentistry, School of Dentistry, Federal University of Rio Grande do Sul, UFRGS, \\ Porto Alegre, Brazil; ' Department of Preventive, Restorative and Pediatric Dentistry, School of Dental Medicine, \\ University of Bern, Bern, Switzerland
}

\section{Key Words}

Caries detection - Demineralization - Dental caries - Laser

fluorescence $\cdot$ Remineralization

\begin{abstract}
This study aimed to evaluate the effectiveness of fluorescence-based methods (DIAGNOdent, LF; DIAGNOdent pen, LFpen, and VistaProof fluorescence camera, FC) in detecting demineralization and remineralization on smooth surfaces in situ. Ten volunteers wore acrylic palatal appliances, each containing 6 enamel blocks that were demineralized for 14 days by exposure to a $20 \%$ sucrose solution and 3 of them were remineralized for 7 days with fluoride dentifrice. Sixty enamel blocks were evaluated at baseline, after demineralization and 30 blocks after remineralization by two examiners using LF, LFpen and FC. They were submitted to surface microhardness (SMH) and cross-sectional microhardness analysis. The integrated loss of surface hardness $(\triangle K H N)$ was calculated. The intraclass correlation coefficient for interexaminer reproducibility ranged from 0.21 (FC) to 0.86 (LFpen). $\mathrm{SMH}, \mathrm{LF}$ and LFpen values presented significant differences among the three phases. However, FC fluorescence values showed no significant differences between the demineral-
\end{abstract}

ization and remineralization phases. Fluorescence values for baseline, demineralized and remineralized enamel were, respectively, $5.4 \pm 1.0,9.2 \pm 2.2$ and $7.0 \pm 1.5$ for LF; $10.5 \pm 2.0$, $15.0 \pm 3.2$ and $12.5 \pm 2.9$ for LFpen, and $1.0 \pm 0.0,1.0 \pm 0.1$ and $1.0 \pm 0.1$ for FC. SMH and $\triangle K H N$ showed significant differences between demineralization and remineralization phases. There was a negative and significant correlation between SMH and LF and LFpen in the remineralization phase. In conclusion, LF and LFpen devices were effective in detecting demineralization and remineralization on smooth surfaces provoked in situ.

(c) 2014 S. Karger AG, Basel

The early detection of smooth surface caries lesions is important to provide proper and noninvasive management; lesions at this stage have the potential to be remineralized and can be monitored over time [Diniz et al., 2009]. Conventional methods for caries detection are not capable of quantifying the mineral loss or gain occurring as a result of demineralization and remineralization processes, respectively [Spiguel et al., 2009].

In this context, quantitative methods have been developed for caries detection and for monitoring changes in

\begin{tabular}{ll}
\hline KARGER & $\begin{array}{l}\text { @ 2014 S. Karger AG, Basel } \\
0008-6568 / 14 / 0486-0507 \$ 39.50 / 0 \quad \text { Karger }\end{array}$ \\
E-Mail karger@karger.com & $\begin{array}{l}\text { This is an Open Access article licensed under the terms of the } \\
\text { Creative Commons Attribution-NonCommercial 3.0 Un- } \\
\text { ported license (CC BY-NC) (www.karger.com/OA-license), } \\
\text { applicable to the online version of the article only. Distribu- } \\
\text { tion permitted for non-commercial purposes only. }\end{array}$
\end{tabular}

Dr. Michele Baffi Diniz

Universidade Cruzeiro do Sul, Setor de Pós Graduação

Rua Galvão Bueno 868

São Paulo, SP 01506-000 (Brazil)

E-Mail mibdiniz@ hotmail.com 
the mineral content [Pretty and Maupomé, 2004]. Some of these methods are based on the fluorescence phenomenon emitted by bacterial porphyrins (fluorophores), molecules that are excited by a light source with a specific excitation wavelength [Hibst et al., 2001; Bader and Shugars, 2004].

The laser fluorescence devices DIAGNOdent (LF; DIAGNOdent 2095, KaVo, Biberach, Germany) and DIAGNOdent pen (LFpen; DIAGNOdent 2190, KaVo) are able to capture, analyze and quantify the fluorescence emitted from bacterial porphyrins and other chromophores [Hibst et al., 2001]. Some studies have evaluated the performance of the LF and LFpen devices in detecting or monitoring caries development on smooth surfaces, with contradictory results [Hibst et al., 2001; Shi et al., 2001a, b; Pinelli et al., 2002; Mendes and Nicolau, 2004; Mendes et al., 2005; Aljehani et al., 2006, 2007], and in monitoring the remineralization process [Mendes et al., 2003; Andersson et al., 2004; Ferreira et al., 2008; Diniz et al., 2009; Kiertsman et al., 2009; Spiguel et al., 2009].

The intraoral fluorescence camera (FC; VistaProof, Dürr Dental, Bietigheim-Bissingen, Germany) was developed for caries detection and emits blue light at $405 \mathrm{~nm}$ and captures fluorescent images from dental surfaces [Rodrigues et al., 2008]. In initial carious lesions, red porphyrin fluorescence is emitted whereas it is absent in sound enamel [Thoms, 2006]. However, there is no scientific evidence available about the cutoff limits used to determine caries lesions on smooth and occlusal surfaces. An in vitro study has shown good reliability in detecting caries on occlusal and smooth surfaces, similar to the LF and LFpen devices [De Benedetto et al., 2011].

To our knowledge, only two studies have evaluated the LF device to detect caries-like lesions created in in situ conditions [Kiertsman et al., 2009; Spiguel et al., 2009]. Furthermore, to date no study has evaluated the effectiveness of LFpen and FC to monitor the de-/remineralization process on smooth surfaces. For that reason, it is relevant to verify the ability of the fluorescence-based methods to provide accurate and reliable measurements to monitor the development and regression of incipient caries lesions.

Therefore, the aim of this study was to evaluate the effectiveness of fluorescence-based methods (LF, LFpen and FC) in detecting demineralization and remineralization provoked on smooth surfaces in situ. The null hypothesis is that there is no difference among the fluorescence-based methods in differentiating demineralization and remineralization on smooth surfaces.

\section{Materials and Methods}

Ethical Aspects

This study was conducted in accordance with the Declaration of Helsinki and it was approved by the Ethics in Research Committee of Cruzeiro do Sul University (204/2011), São Paulo, Brazil. The study aim, procedures, possible discomforts and risks, safety, and benefits were fully explained to the subjects. Informed consent was obtained from all volunteers prior to the investigation.

\section{Experimental Design}

This in situ study involved three phases performed over 21 days: baseline (I), demineralization (II) and remineralization (III).

\section{Baseline}

Specimen Preparation

One hundred enamel blocks $(4 \times 4 \times 2 \mathrm{~mm})$ were obtained from bovine incisors and were stored in $0.1 \%$ thymol solution $(\mathrm{pH} 7.0)$ at room temperature. Each block was embedded in epoxy resin in order to expose only the buccal surface. This procedure is needed for an appropriate surface microhardness (SMH) analysis [Diniz et al., 2009].

The blocks were then stored individually at $100 \%$ humidity. Afterwards, the enamel surface was serially polished with carbide paper $(600,1,200$ and 1,500 grid, in sequence; Buehler, Lake Bluff, Ill., USA) and diamond abrasive on a polishing paper, resulting in removal of about $100 \mu \mathrm{m}$ of the outer enamel, which was controlled with a micrometer. SMH analysis was performed using a microhardness tester (HMV-2; Shimadzu Corp., Tokyo, Japan) with a Knoop diamond under a 25-gram load for $5 \mathrm{~s}$ [Spiguel et al., 2009]. Five indentations spaced $100 \mu \mathrm{m}$ from each other were made and the average was recorded. From 100 enamel blocks, only 60 with a hardness of $351.1 \pm 18.0 \mathrm{KHN}$ were selected. Enamel blocks were sterilized with gamma radiation (25 kGy).

\section{Measurements with Fluorescence-Based Methods}

Each enamel block was assessed by two examiners using LF and LFpen devices and FC. The examiners have experience in using and handling the devices, since they had participated in previous studies [Rodrigues et al., 2008; Diniz et al., 2009, 2012]. The enamel blocks were removed from the $100 \%$ humidity storage environment, fixed in clear acrylic resin disks and dried with a paper tissue [Spiguel et al., 2009].

The LF and LFpen measurements were performed using a fiber-optic conical tip (tip B), specifically designed for smooth surfaces, and the cylindrical sapphire fiber tip, respectively, according to the manufacturer's instructions. Before each measurement, the devices were calibrated against a ceramic standard, and then they were recalibrated after testing 10 blocks [Mendes et al., 2003; Spiguel et al., 2009]. After calibration, the laser point was placed in the center of each enamel block and swept across the surface. The maximum fluorescence value detected by the devices was recorded. Each block was dried with a paper tissue and air-dried for $5 \mathrm{~s}$ and analyzed 3 times in sequence by each examiner and the mean values were calculated [Diniz et al., 2009].

The FC measurements were performed in a dark environment. After capturing the images of the enamel blocks, they were analyzed by FC-specific software (DBSWIN, Dürr Dental), which translates the red and green rate of fluorescence into numbers that correspond to the lesion severity [Rodrigues et al., 2008]. The val- 
ues were recorded for further analysis. The FC measurements were also done 3 times by each examiner and the mean values were calculated.

\section{Participant Selection}

Ten volunteers ( 4 males and 6 females, aged 20-30 years) who lived in an area whose water supply contained fluoride $(0.7 \mathrm{mg} \mathrm{F} / \mathrm{l})$ were selected for the investigation. They were in good general and oral health and presented a normal salivary flow rate. The volunteers were clinically evaluated after professional dental prophylaxis for the detection of active caries lesions and periodontal disease. The participants were willing to cooperate with the clinical research protocol and to abstain from their own oral hygiene products, except those provided for this study. The exclusion criteria were current or recent use of any form of medication that affects salivary flow, use of fixed or removable orthodontic appliances, dental treatment, presence of active caries lesions or periodontal disease, pregnant or breast-feeding, smoker, or systemic illness [Spiguel et al., 2009].

A meeting was organized with the volunteers in order to present the research project, its objectives and the experimental design. The volunteers received oral and written information regarding the procedures to be performed during the experiment and to refrain from using any antibacterial or fluoridated product.

\section{Demineralization Phase}

During this phase, the volunteers wore intraoral acrylic palatal appliances. Six spaces were created and one sterilized enamel block was placed in each, leaving a $1.0-\mathrm{mm}$ space for plaque accumulation. Dental plaque was formed on the enamel blocks, which were protected from mechanical disturbance by a plastic mesh fixed in the acrylic surface [Cury et al., 2000].

The volunteers were instructed to remove the intraoral appliance 8 times per day for 14 days and to place 2 drops of $20 \%$ sucrose solution onto each enamel block (at 8.00, 10.00, 12.00, 14.00, 16.00, $18.00,20.00$ and 22.00) [Spiguel et al., 2009]. The appliance was placed back into the mouth $5 \mathrm{~min}$ after each cariogenic challenge. Volunteers were instructed to wear the appliances continuously, including at night, except during meals, when drinking water, when consuming any acidic beverage or during oral care [Aires et al., 2006]. They brushed their natural teeth with nonfluoride dentifrice (Cocoricó, Bitufo, Itupeva, São Paulo, Brazil) [Spiguel et al., 2009].

After the demineralization phase, the enamel blocks $(n=60)$ were removed from the appliances and gently brushed. SMH and fluorescence-based measurements were obtained. For SMH analysis, 5 indentations spaced $100 \mu \mathrm{m}$ from each other and from the baseline indentations were made [Vieira et al., 2005]. Afterwards, 30 enamel blocks ( 3 enamel blocks from each volunteer) were selected randomly for cross-sectional microhardness (CSMH) analysis and polarized light microscopy. A 7-day period was allowed between demineralization and remineralization phases to allow the examinations. Francescut et al. [2006] showed that the fluorescence values significantly decreased only after 7-14 days of storage.

\section{Remineralization Phase}

The other 30 enamel blocks were rinsed in deionized water and replaced in each appliance without the plastic mesh protection. The volunteers wore the intraoral appliances again for 7 days and were instructed to wear them all the time, including at night, but to remove them during meals ( $1 \mathrm{~h}$ per meal) [Spiguel et al., 2009].

They were instructed to perform oral hygiene 3 times a day and the appliances were to be brushed in the mouth, including the enamel blocks, using fluoridated dentifrice (Colgate Total 12 Professional Gengiva Saudável, Colgate-Palmolive Ind. E Com. Ltda., São Paulo, Brazil, 1,450 $\mu \mathrm{g} \mathrm{F/g}$ ) for $2 \mathrm{~min}$. After the remineralization phase, $\mathrm{SMH}$ and fluorescence-based measurements were obtained again. For SMH analysis, 5 indentations spaced $100 \mu \mathrm{m}$ from each other and $200 \mu \mathrm{m}$ from the baseline indentations were made [Vieira et al., 2005]. CSMH analysis and polarized light microscopy were performed.

\section{Cross-Sectional Microhardness}

After SMH analysis, all enamel blocks were longitudinally sectioned through the center of the exposed enamel for CSMH determination. Half of each block was embedded in acrylic resin and the cut surfaces were exposed and polished. The CSMH was performed according to Spiguel et al. [2009] using a Knoop indenter with a 25-gram load for $5 \mathrm{~s}$ (Shimadzu HMV-2). Three rows of 8 indentations at 10, 30, 50, 70, 90, 110, 220 and $330 \mu \mathrm{m}$ from the outer enamel surface were made: one row in the central region of the exposed enamel and the other two spaced $100 \mu \mathrm{m}$ from the first. The mean value of each distance was calculated.

Integrated hardness $(\mathrm{KHN} \times \mu \mathrm{m})$ of sound, demineralized and remineralized enamel was calculated to a depth of $220 \mu \mathrm{m}$ using the trapezoidal rule [Cury et al., 2000] (GraphPad Prism, version 3.02; GraphPad Software Inc., La Jolla, Calif., USA). The integrated loss of hardness $(\triangle \mathrm{KHN})$ was calculated by subtracting the integrated hardness (demineralized or remineralized) from the integrated hardness of sound enamel [Spiguel et al., 2009].

\section{Polarized Light Microscopy}

The other half of the enamel block was sectioned to approximately $500 \mu \mathrm{m}$ thickness using a diamond saw. The sections were then manually ground and polished to a thickness of $100 \mu \mathrm{m}$, mounted on slides with distilled/deionized water and covered with a glass coverslip. The sections were examined by polarized light microscopy (Leica DM750, Leica Microsystems, Wetzlar, Germany) at $\times 400$ magnification. Three areas in the central region of the sections were analyzed by recording the thickness of the superficial enamel layer and the depth of the lesion using Image $1.38 \mathrm{x}$ software (National Institutes of Health, USA) [Spiguel et al., 2009].

\section{Statistical Analysis}

The data were analyzed using the statistical software MedCalc for Windows (version 12.3.0, Mariakerke, Belgium), and the level of significance was $\mathrm{p}<0.05$. Outcome variables were the mean values of LF, LFpen, FC, SMH, integrated hardness and $\triangle \mathrm{KHN}$, and the phases (baseline, demineralization and remineralization) as variation factors.

The intraclass correlation coefficient (ICC) was used to assess interexaminer reproducibility for fluorescence-based methods. The ICC was considered poor when the values were below 0.40 , fair for values between 0.40 and 0.59 , good for values between 0.60 and 0.75 , and excellent for values above 0.75 [Lin, 1989].

The percentage change of surface microhardness (\%SMHC), determined in relation to the baseline measurement, was calculated for each enamel block according to the method of Cury et al. [2000]: \%SMHC $=($ SMH after demineralization - baseline $\times 100) /$ 
Table 1. ICC for interexaminer reproducibility for LF, LFpen and FC in all phases ( $\mathrm{n}=10$ volunteers)

\begin{tabular}{llll}
\hline \multirow{2}{*}{ Phases } & \multicolumn{2}{l}{ ICC $(95 \%$ confidence interval $)$} & \\
\cline { 2 - 4 } & LF & LFpen & FC \\
\hline Baseline & $0.60(0.33-0.76)$ & $0.45(0.09-0.67)$ & $0.21(-0.33$ to 0.53$)$ \\
Demineralization & $0.70(0.49-0.82)$ & $0.86(0.76-0.92)$ & $0.47(0.12-0.68)$ \\
Remineralization & $0.77(0.52-0.89)$ & $0.51(-0.03$ to 0.77$)$ & $0.68(0.33-0.85)$ \\
\hline
\end{tabular}

Table 2. SMH values, $\% \mathrm{SMHC}, \% \mathrm{SMR}$, integrated hardness $(\mathrm{KHN} \times \mu \mathrm{m})$ and $\triangle \mathrm{KHN}(\mathrm{mean} \pm$ standard deviation) in all phases ( $\mathrm{n}=10$ volunteers)

\begin{tabular}{llllll}
\hline Phases & SMH, KHN & \%SMHC & \%SMR & $\begin{array}{l}\text { Integrated hardness, } \\
\text { KHN } \times \mu \mathrm{m}\end{array}$ & $\Delta \mathrm{KHN}$ \\
\hline Baseline & $351.1 \pm 18.0^{\mathrm{a}}$ & - & - & $36,940 \pm 861^{\mathrm{a}}$ & - \\
Demineralization & $113.7 \pm 69.4^{\mathrm{b}}$ & $-67.8 \pm 18.9^{\mathrm{a}}$ & - & $19,160 \pm 1,553^{\mathrm{b}}$ & $17,780 \pm 3,544^{\mathrm{a}}$ \\
Remineralization & $196.4 \pm 85.9^{\mathrm{c}}$ & $-43.9 \pm 25.2^{\mathrm{b}}$ & $36.6 \pm 35.5$ & $29,993 \pm 1,883^{\mathrm{c}}$ & $6,947 \pm 3,738^{\mathrm{b}}$ \\
\hline
\end{tabular}

Significant differences $(\mathrm{p}<0.05)$ are indicated within the same column by different superscript letters.

Table 3. Fluorescence values (mean \pm standard deviation) for LF, LFpen and FC in all phases ( $\mathrm{n}=10$ volunteers)

\begin{tabular}{llll}
\hline Phases & LF & LFpen & FC \\
\hline Baseline & $5.4 \pm 1.0^{\mathrm{a}}$ & $10.5 \pm 2.0^{\mathrm{a}}$ & $1.0 \pm 0.0^{\mathrm{a}}$ \\
Demineralization & $9.2 \pm 2.2^{\mathrm{b}}$ & $15.0 \pm 3.2^{\mathrm{b}}$ & $1.0 \pm 0.1^{\mathrm{b}}$ \\
Remineralization & $7.0 \pm 1.5^{\mathrm{c}}$ & $12.5 \pm 2.9^{\mathrm{c}}$ & $1.0 \pm 0.1^{\mathrm{b}}$ \\
\hline
\end{tabular}

Significant differences $(\mathrm{p}<0.05)$ are indicated within the same column by different superscript letters.

baseline or $($ SMH after remineralization - baseline $\times 100)$ /baseline. The percentage of enamel surface microhardness recovery (\%SMR) was calculated for each enamel block remineralized according to the method of Cury et al. [2005]: \%SMR $=[100(\mathrm{SMH}$ post-treatment - SMH caries)/baseline $\mathrm{SMH}-\mathrm{SMH}$ caries].

In order to compare the three phases of the experiment for $\mathrm{SMH}$, fluorescence-based measurements and integrated hardness, the nonparametric Friedman's and multiple comparison tests were performed. The Mann-Whitney test was used to compare the $\% \mathrm{SMHC}$ and $\triangle \mathrm{KHN}$ for demineralization and remineralization phases.

Spearman's rank correlation coefficient (rho) was used to test the strength of a relationship between the different fluorescencebased methods and $\mathrm{SMH}$ or $\triangle \mathrm{KHN}$, considering all phases of the experiment. The Spearman coefficient varies between -1 and 1 ; the closer these extremes, the greater is the association between variables.

\section{Results}

Table 1 presents the interexaminer reproducibility assessed by calculating the ICC values for LF, LFpen and FC in all phases, ranging from poor to excellent agreement between the examiners.

With regard to the $\mathrm{SMH}$ analysis, statistically significant differences were observed among the three phases $(\mathrm{p}<0.05)$. The \%SMHC was also significantly different between demineralization and remineralization phases $(\mathrm{p}<0.05)$. The $\%$ SMR was $36.6 \pm 35.0$. Integrated hardness was significantly different among all phases $(\mathrm{p}<0.05)$. $\triangle \mathrm{KHN}$ was also significantly different between demineralization and remineralization phases $(\mathrm{p}<0.05$, table 2$)$.

Table 3 presents the mean fluorescence values for LF, LFpen and FC in all phases. The LF and LFpen measurements showed significant differences among the three phases, with the highest values obtained for the demineralization phase $(\mathrm{p}<0.05)$. With respect to the FC measurements, there was no difference between demineralization and remineralization phases $(\mathrm{p}>0.05)$; however, those phases presented significant differences compared to baseline.

Spearman's rank correlation coefficients are shown in table 4. There was a negative correlation between SMH/ $\triangle \mathrm{KHN}$ and fluorescence values in all phases, with a statistically significant difference in the remineralization 
Table 4. Spearman's rank correlation coefficients between fluorescence-based values and SMH and $\Delta \mathrm{KHN}$ in all phases ( $\mathrm{n}=10$ volunteers)

\begin{tabular}{|c|c|c|c|c|c|c|}
\hline \multirow[t]{3}{*}{ Phases } & \multicolumn{6}{|c|}{ Spearman correlation coefficient ( $\mathrm{p}$ value) } \\
\hline & \multicolumn{3}{|l|}{$\mathrm{SMH}$} & \multicolumn{3}{|l|}{$\Delta \mathrm{KHN}$} \\
\hline & LF & LFpen & $\mathrm{FC}$ & LF & LFpen & FC \\
\hline Baseline & $-0.155(\mathrm{p}=0.2370)$ & $-0.062(\mathrm{p}=0.6395)$ & $-0.187(\mathrm{p}=0.1526)$ & - & - & - \\
\hline Demineralization & $-0.075(\mathrm{p}=0.5675)$ & $-0.030(\mathrm{p}=0.8203)$ & $-0.151(\mathrm{p}=0.2499)$ & $-0.107(\mathrm{p}=0.4166)$ & $-0.087(\mathrm{p}=0.5088)$ & $-0.160(\mathrm{p}=0.2228)$ \\
\hline Remineralization & $-0.339 *(\mathrm{p}=0.0081)$ & $-0.445^{*}(\mathrm{p}=0.0004)$ & $-0.166(\mathrm{p}=0.1877)$ & $-0.167(\mathrm{p}=0.2023)$ & $-0.180(\mathrm{p}=0.1694)$ & $-0.057(\mathrm{p}=0.6658)$ \\
\hline
\end{tabular}

Variables statistically correlated: ${ }^{*} \mathrm{p}<0.05$

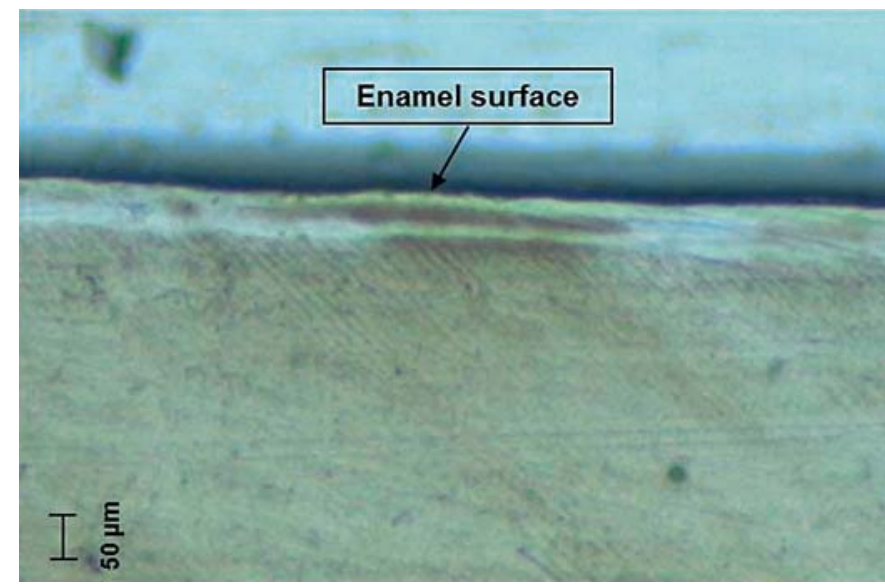

Fig. 1. Polarized light micrograph of an enamel block after demineralization. $\times 400$.

phase $(\mathrm{p}<0.05)$ for LF and LFpen measurements and $\mathrm{SMH}$. The highest correlation was found for LFpen-remineralization $(\mathrm{r}=-0.445)$, which means the higher the SMH values after remineralization, the lower the LF and LFpen measurements.

Figure 1 shows a polarized light photomicrograph after demineralization. The mean lesion depth was $70.6 \pm$ $21.1 \mu \mathrm{m}$ and the thickness of the surface layer was $8.7 \pm$ $2.4 \mu \mathrm{m}$. Spearman's correlation coefficients between fluorescence values and lesion depth after demineralization were low and nonsignificant: $r=0.013$ for $L F, r=0.032$ for LFpen and $\mathrm{r}=0.152$ for FC.

Figure 2 shows a polarized light photomicrograph after remineralization. The mean lesion depth was $35.8 \pm$ $14.3 \mu \mathrm{m}$ and the thickness of the surface layer was $9.9 \pm$ $2.0 \mu \mathrm{m}$. Spearman's correlation coefficients between fluorescence values and lesion depth after remineralization were also nonsignificant: $r=0.234$ for $L F, r=0.278$ for LFpen and $r=0.122$ for FC.

Fluorescence-Based Methods for Monitoring Caries Lesions in situ

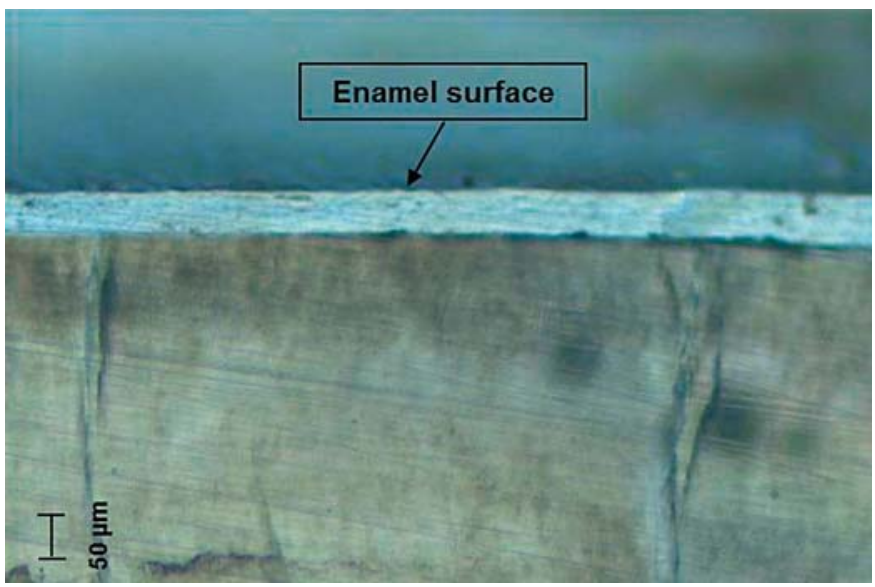

Fig. 2. Polarized light micrograph of an enamel block after remineralization. $\times 400$.

\section{Discussion}

In this study, the effectiveness of fluorescence-based methods was evaluated in the detection of demineralization and remineralization on smooth surfaces. It is important to emphasize that a caries detection method should present good reproducibility, allowing for the acquisition of consistent and reliable results between different evaluations and examiners. According to Lussi and Hellwig [2006], a high level of agreement for the LF device means that it could be useful for monitoring the carious process. In this study, ICC values for interexaminer reproducibility showed great variability among the fluorescence-based methods as well as in all phases of the study. Higher ICC values were observed after demineralization for LFpen and after remineralization for LF, indicating excellent agreement between the examiners. These results corroborate the studies by Aljehani et al. [2007] and De Benedetto et al. [2011], who also observed high reproduc-

Caries Res 2014;48:507-514 
ibility values for LF and LFpen devices for caries detection on smooth surfaces. On the other hand, moderate agreement was found for the LF device at baseline and after demineralization phases, a fact also reported by Diniz et al. [2009]. In general, LFpen was less reproducible than LF. This could be attributed to the difficulty of handling the LFpen device by the different examiners and its more fragile tip made of sapphire fiber.

It was observed that the FC device showed poor agreement between examiners in the baseline phase. This might be due to specimen size and the polishing procedure of the enamel surfaces, which reflected the six-LED light sources and may have interfered with image capture and fluorescence analysis by the different examiners and led to subjective errors during measurements. On the other hand, fair and good agreement was observed in the demineralization and remineralization phases, respectively. This might be explained by the lack of reflection of the six-LED light sources on the opaque surface due to the demineralization process. Controversially, De Benedetto et al. [2011] observed that the FC device showed high reproducibility on smooth surfaces of primary teeth, resembling the LF and LFpen devices. These differences may be explained by the fact that in that study natural caries lesions were assessed on smooth surfaces.

To date, no information about the effectiveness of LFpen and FC devices to monitor the de-/remineralization process on smooth surfaces is available. The FC fluorescence values were statistically significantly different between baseline and demineralization or remineralization phase. However, no difference was found between demineralization and remineralization phases. It could be suggested that FC was not able to identify small changes in enamel mineral content. These results were also found when occlusal dentin lesions were analyzed in previous studies [Rodrigues et al., 2008; Diniz et al., 2012].

In the present study, the LF and LFpen fluorescence values showed significant differences among the three phases, while FC did not. Therefore, the null hypothesis can be rejected. In previous studies, the LF device was suitable for detecting caries on smooth surfaces and it showed a significant increase in fluorescence values after the cariogenic challenge [Mendes and Nicolau, 2004; Ferreira et al., 2008; Spiguel et al., 2009] and between demineralization and remineralization [Spiguel et al., 2009], as was found in the present investigation. Divergent results were found in other studies. Kiertsman et al. [2009] found no statistically significant difference in LF fluorescence values among baseline, demineralization and remineralization of occlusal surfaces of human premolars, which might be due to the complex invaginated anatomy of pits and fissures. Mendes et al. [2003] and Diniz et al. [2009] reported that the LF device was not appropriate for monitoring the demineralization and remineralization processes. This fact could be explained by differences in methodologies, such as the type of dental substrate (human or bovine enamel), natural versus artificial caries lesions and remineralizing agent ( $\mathrm{pH}$-cycling models, fluoride dentifrice or fluoride gel). Since LF detects changes in organic tooth content, such as fluorophores and other chromophores produced by cariogenic bacteria rather than inorganic content [Lussi et al., 1999; Shi et al., 2000; Hibst et al., 2001], its effectiveness is questionable in artificial caries-like enamel lesions, which are induced without metabolites from oral bacteria. The LF and LFpen fluorescence values were significantly greater after demineralization when compared to the baseline values, which might be explained by increase in the surface porosity of the enamel and light scattering [Mendes and Nicolau, 2004] and/or the increase in organic content in the presence of biofilm [Spiguel et al., 2009]. The presence of microorganisms has been shown within active and inactive incipient caries lesions by scanning electron microscopy [Parolo and Maltz, 2006]. Thus, it is possible that the penetration of metabolic products into enamel tissue in early caries lesions, producing significant amounts of endogenous porphyrins and related compounds, results in an increase in fluorescence values.

The fluorescence values obtained using LFpen were higher than those obtained using the LF device in agreement with previous studies [Diniz et al., 2008; Rodrigues et al., 2008; Diniz et al., 2012]. The reasons for this finding could be attributed to the different diameters and materials of the tips in both devices, which might influence the amount of the excitation light transmitted through the tip and the degree of capture of the fluorescence emitted by the dental tissues.

Another important aspect to be discussed is related to the cutoff limits proposed for each device. The performance of fluorescence-based methods is dependent on the cutoff limits used and the difference in cutoff values for sound teeth, enamel or dentine caries will affect the treatment decision-making in clinical practice [HeinrichWeltzien et al., 2003]. It was observed that changes in LF (baseline) and LFpen (baseline, after demineralization and remineralization) fluorescence values were within the cutoff limits proposed by the manufacturer. Although the manufacturer indicates the cutoff values for all surfaces, they were based on studies assessing occlusal lesions. This should be considered when LF devices are 
used in clinical practice on smooth surfaces. In the present study, the FC values obtained in all phases were very close to each other, ranging from sound to demineralized when classified according to the cutoff limits proposed by the manufacturer, making it difficult to monitor incipient caries lesions. These results suggest that care should be taken in the use of the cutoffs, since there is no consensus in the literature for active and inactive incipient caries lesions on smooth surfaces. Thus, fluorescence-based methods should be used as complementary methods in monitoring incipient caries lesions on smooth surfaces.

There was a negative and moderate correlation between SMH and fluorescence values (LF and LFpen) in the remineralization phase $(\mathrm{p}<0.05)$. This means that when there was an increase in SMH values, indicating remineralization, fluorescence values decreased. These results show that LF and LFpen devices might be able to detect the remineralization process of early enamel lesions, which differs from the results described for the LF device by Spiguel et al. [2009]. However, there was no significant correlation between fluorescence values and lesion depth, which means that fluorescence might be related to the softening of the surface rather than to subsurface demineralization.

The lack of correlation between hardness and LF and LFpen for demineralization can be explained by the fact that the devices were not capable of detecting small changes in mineral content due to the scattering phenomenon [Shi et al., 2001a; Mendes et al., 2003], which might be due to the porosity of caries lesions [Mendes and Nicolau, 2004; Diniz et al., 2009]. However, there was a significant correlation for LF and LFpen after the remineralization process when slight surface hardness recovery was obtained and LF values increased, which might be related to the decrease in porosity and the scattering phenomenon. The absence of correlation between FC and hardness for any phase could be attributed to the small range in the cutoff limits of the device for sound and enamel caries [Rodrigues et al., 2008; Diniz et al., 2012].
It is important to discuss the cost-benefit ratio of the fluorescence-based methods, which should be considered as adjunct tools to visual examination for caries detection and monitoring on smooth surfaces, when bitewing radiographs are not indicated. Fluorescence values should be carefully interpreted in the treatment decision-making in clinical practice. To date, studies regarding the efficacy of fluorescence-based methods in monitoring the caries process are limited, especially with respect to LFpen and FC devices on smooth surfaces. Moreover, clinical studies should be performed in order to evaluate the effectiveness of the fluorescence-based methods in monitor demineralization and remineralization processes.

It is reasonable to conclude that LF and LFpen devices were effective in differentiating demineralization and remineralization on smooth surfaces in situ, with moderate correlation with SMH for the remineralization of enamel. However, the results should not be considered as indicating exact threshold measurements. Besides, FC was not able to differentiate between demineralization and remineralization phases.

\section{Acknowledgments}

The authors are grateful to all volunteers. They wish to thank the Coordenação de Aperfeiçoamento de Pessoal de Nível Superior (CAPES) and the Fundação de Amparo à Pesquisa do Estado de São Paulo (FAPESP - protocol 2010/06855-0) for their financial support. This study was submitted to Cruzeiro do Sul University as a requirement for the master's degree in pediatric dentistry.

\section{Disclosure Statement}

The authors declare that they have no proprietary, financial, professional or other personal interest of any nature or kind in any product, service and/or company that might introduce bias or affect their judgment or that could be construed as influencing the position presented herein or the review of the manuscript entitled 'Effectiveness of Fluorescence-Based Methods to Detect in situ Demineralization and Remineralization on Smooth Surfaces'.
References

Fluorescence-Based Methods for

Monitoring Caries Lesions in situ

\footnotetext{
Aires CP, Tabchoury CPM, Del Bel Cury AA, Koo $\mathrm{H}$, Cury JA: Effect of sucrose concentration on dental biofilm formed in situ and on enamel demineralization. Caries Res 2006;40: 28-32.

Aljehani A, Bamzahim M, Yousif MA, Shi XQ: In vivo reliability of an infrared fluorescence method for quantification of carious lesions in orthodontic patients. Oral Health Prev Dent 2006;4:145-150.
}

\footnotetext{
Aljehani A, Yang L, Shi XQ: In vitro quantification of smooth surface caries with DIAGNOdent and the DIAGNOdent pen. Acta Odontol Scand 2007;65:60-63.

Andersson A, Sköld-Larsson K, Petersson L, Twetman S: Measurement of enamel lesion regression with a laser fluorescence device (DIAGNOdent): a pilot study. Orthodontics 2004;1:201-205.
} 
Bader JD, Shugars DA: A systematic review of the performance of a laser fluorescence device for detecting caries. J Am Dent Assoc 2004;135: 1414-1426.

-Cury JA, Rebelo MAB, Del Bel Cury AA, Derbyshire MTVC, Tabchoury CPM: Biomechanical composition and cariogenicity of dental plaque formed in the presence of sucrose or glucose and fructose. Caries Res 2000;34:491-497.

-Cury JA, Simões GS, Del Bel Cury AA, Gonçalves NC, Tabchoury CP: Effect of a calcium carbonate-based dentifrice on in situ enamel remineralization. Caries Res 2005;39:255257.

-De Benedetto MS, Morais CC, Novaes TF, de Almeida Rodrigues J, Braga MM, Mendes FM: Comparing the reliability of a new fluorescence camera with conventional laser fluorescence devices in detecting caries lesions in occlusal and smooth surfaces of primary teeth. Lasers Med Sci 2011;26:157-162.

-Diniz MB, Boldieri T, Rodrigues JA, Santos-Pinto L, Lussi A, Cordeiro RC: The performance of conventional and fluorescence-based methods for occlusal caries detection: an in vivo study with histologic validation. J Am Dent Assoc 2012;143:339-350.

Diniz MB, Paes Leme AF, Cardoso KS, Rodrigues JA, Cordeiro RCL: The efficacy of laser fluorescence to detect in vitro demineralization and remineralization of smooth enamel surfaces. Photomed Laser Surg 2009;27:57-61.

- Diniz MB, Rodrigues JA, Hug I, Cordeiro RCL, Lussi A: The influence of pit and fissure sealants on infrared fluorescence measurements. Caries Res 2008;42:328-333.

Ferreira JM, Silva MF, Oliveira AF, Sampaio FC: Evaluation of different methods for monitoring incipient carious lesions in smooth surfaces under fluoride varnish therapy. Int J Paediatr Dent 2008;18:300-305.
Francescut P, Zimmerli B, Lussi A: Influence of different storage methods on laser fluorescence values: a two-year study. Caries Res 2006; 40:181-185.

Heinrich-Weltzien R, Kühnisch J, Oehme T, Ziehe A, Stösser L, García-Godoy F: Comparison of different DIAGNOdent cut-off limits for in vivo detection of occlusal caries. Oper Dent 2003;28:672-680.

-Hibst R, Paulus R, Lussi A: A detection of occlusal caries by laser fluorescence: basic and clinical investigations. Med Laser Applic 2001;16: 295-213.

Kiertsman F, Camargo LB, Bonifácio CC, Imparato JCP, Raggio DP, Mendes FM: Effectiveness of laser fluorescence device in monitoring demineralization and remineralization of occlusal caries lesions - in situ study. Rev Inst Ciênc Saúde 2009;27:384-389.

Lin LIK: A concordance correlation coefficient to evaluate reproducibility. Biometrics 1989;45: 255-268.

Lussi A, Hellwig E: Performance of a new laser fluorescence device for the detection of occlusal caries in vitro. J Dent 2006;34:467-471.

Lussi A, Imwinkelried S, Pitts N, Longbottom C Reich E: Performance and reproducibility of a laser fluorescence system for detection of occlusal caries in vitro. Caries Res 1999;33: 261-266.

Mendes FM, Nicolau J: Utilization of laser fluorescence to monitor caries lesions development in primary teeth. J Dent Child 2004;71: 139-142.

Mendes FM, Nicolau J, Duarte DA: Evaluation of the effectiveness of laser fluorescence in monitoring in vitro remineralization of incipient caries lesions in primary teeth. Caries Res 2003;37:442-444.

Mendes FM, Siqueira WL, Mazzitelli JF, Pinheiro SL, Bengtson AL: Performance of DIAGNOdent for detection and quantification of smooth-surface caries in primary teeth. J Dent 2005;33:79-84.
- Parolo CCF, Maltz M: Microbial contamination of noncavitated caries lesions: a scanning electron microscopic study. Caries Res 2006; 40:536-541.

Pinelli C, Campos Serra M, de Castro Monteiro Loffredo L: Validity and reproducibility of a laser fluorescence system for detecting the activity of white-spot lesions on free smooth surfaces in vivo. Caries Res 2002;36:19-24.

Pretty IA, Maupomé G: A closer look at diagnosis in clinical dental practice. Part 5: Emerging technologies for caries detection and diagnosis. J Can Dent Assoc 2004;70:540, 540a-540i.

Rodrigues JA, Hug I, Diniz MB, Lussi A: Performance of fluorescence methods, radiographic examination and ICDAS II on occlusal surfaces in vitro. Caries Res 2008;42:297-304.

-Shi XQ, Tranaeus S, Angmar-Mansson B: Validation of DIAGNOdent for quantification of smooth-surface caries: an in vitro study. Acta Odontol Scand 2001a;59:74-78.

- Shi XQ, Tranaeus S, Angmar-Mansson B: Comparison of QLF and DIAGNOdent for quantification of smooth surface caries. Caries Res 2001b;35:21-26.

-Shi XQ, Welander U, Angmar-Mansson B: Occlusal caries detection with KaVo DIAGNOdent and radiography: an in vitro comparison. Caries Res 2000;34:151-158.

- Spiguel MH, Tovo MF, Kramer PF, Franco KS, Alves KMRP, Delbem ACB: Evaluation of laser fluorescence in the monitoring of the initial stage of the de-/remineralization process: an in vitro and in situ study. Caries Res 2009; 43:302-307.

Thoms M: Detection of intraoral lesions using a fluorescence camera. Proc SPIE Lasers in Dentistry XII 2006;6137:1-7.

-Vieira AEM, Delbem ACB, Sassaki KT, Rodrigues E, Cury JA, Cunha RF: Fluoride dose response in $\mathrm{pH}$-cycling models using bovine enamel. Caries Res 2005;39:514-520. 